\title{
Fuzzy Soft Sets and Fuzzy Soft Lattices
}

\author{
Yingchao Shao* Keyun Qin \\ School of Mathematics Southwest Jiaotong University, Chengdu, Sichuan, 610031, China, \\ *E-mail: shaoyingchao@sina.com(Shao),keyunqin@263.net(Qin) \\ Received 11 September 2011 \\ Accepted 31 July 2012
}

\begin{abstract}
The theory of soft sets was introduced by Molodtsov in 1999 in order to deal with uncertainties. In this paper, the notion of fuzzy soft lattice is defined and some related properties are derived, which extends the notion of a fuzzy lattice to include the algebraic structures of soft sets. Then the lattice structure of fuzzy soft lattices are discussed. At last, the concept of fuzzy soft ideal over a lattice is presented and the lattice structures of fuzzy soft ideal over a lattice are discussed.
\end{abstract}

Keywords: Soft set; Fuzzy soft set; Fuzzy soft lattice; Lattice

\section{Introduction}

Most of the problems in engineering, medical science, economics, environments etc. have various uncertainties. To overcome these uncertainties, some kinds of theories were given which we can use as mathematical tools for dealing with uncertainties. However, these theories have their own difficulties. In 1999, Molodtsov ${ }^{1}$ introduced the notion of soft set to deal with uncertainty.

From then on, works on the soft set theory are progressing rapidly. Soft set theory has a rich potential for applications in several directions, few of which had been shown by Molodtsov ${ }^{1}$. Maji et al. ${ }^{2}$ described the application of soft set theory to a decision making problem. Chen et al. ${ }^{3}$ presented a new definition of soft set parametrization reduction, and compared this definition to the related concept of attributes reduction in rough set theory. In theoretical aspects, Maji et al. $^{4}$ defined several operations on soft sets and made a theoretical study on the theory of soft sets. Qin and Hong ${ }^{5}$ constructed the lat- tice structures of soft sets and introduced the concept of soft equality. H.Aktas and N.Cağman ${ }^{6}$ proposed a definition of soft groups and derived their basic properties. Feng et al. ${ }^{7}$ initiated the study of soft semirings, soft ideals on soft semirings and idealistic soft semirings. Sun et al. ${ }^{8}$ defined the concept of soft modules and studied their basic properties. Furthermore, Ali et al. ${ }^{9}$ introduced some new operations on soft sets and improved the notion of complement of soft set. Jun ${ }^{10}$ applied the notion of soft sets to the theory of $B C K / B C I$-algebras and introduced the notions of soft $B C K / B C I$-algebras and soft subalgebras and derived their basic properties. By means of $\epsilon$-soft and $q$-soft sets, Zhan and Jun ${ }^{11}$ investigated some characterizations of (implicative, positive implicative and fantastic) filteristic soft $B L$ algebras and proved that a soft set is an implicative filteristic soft $B L$-algebra if and only if it is both a positive implicative filteristic soft $B L$-algebra and a fantastic filteristic soft $B L$-algebra.

Fuzzy set theory was initiated by $Z_{\text {adeh }}{ }^{12}$ in 1965. Maji et al. ${ }^{4}$ and Majudar ${ }^{13}$ introduced the

\footnotetext{
${ }^{*}$ Corresponding address:School of Mathematics Southwest Jiaotong University, Chengdu, Sichuan, 610031, China. Tel:15008231457.
} 
concept of fuzzy soft set, a more generalised concept, which is a combination of fuzzy set and studied its properties, respectively. Roy and Maji ${ }^{14}$ presented some applications of this notion to decision making problems. Later on, Kong et al. ${ }^{15,16}$ applied the soft set theoretic approach in decision making problems. Majumdar and Samanta ${ }^{17,18}$ studied the problem of similarity measurement between soft sets and fuzzy soft sets. Yang ${ }^{19}$ presented the notions of fuzzy soft semigroup and fuzzy soft ideal and discussed fuzzy soft image and fuzzy soft inverse image of fuzzy soft semigroup. Aygünoğlu and Aygün ${ }^{20}$ introduced the concept of fuzzy soft group and discussed and studied some of their properties and structural characteristics.

In the first half of the nineteenth century, G.Boole' attempt to formalize propositional logic led to the concept of Boolean algebras. G. Birkhoff's work in 1930s started the general development of lattice theory ${ }^{21}$. The lattice theory has been applied to many kinds od fields. Recently, the work introducing the soft set theory to the lattice theory and the fuzzy set theory have been initiated. $\mathrm{Fu}^{22}$ presented the notion of the soft lattice and derived the properties of the soft lattices and discussed the relationship between the soft lattices and the fuzzy soft sets. Marudai and Rajendran ${ }^{23}$ studied the notion of soft set and fuzzy soft set considering the fact that the parameters are mostly fuzzy hedges or fuzzy parameters. They introduced the notion of fuzzy soft lattice on groups, homomorphic image, pre-image of fuzzy soft lattices, arbitrary family of fuzzy soft lattices and fuzzy normal soft lattices using T-norms. They also investigated the notion of sensible fuzzy soft lattices in groups and some related properties on it. In this paper, we apply the notion of fuzzy soft sets introduced by Maji et al. ${ }^{4}$ to the lattice theory and present the notion of fuzzy soft lattice, which is different from that presented by Marudai et al. ${ }^{23}$, then derive their basic properties and investigate the algebraic structure of the fuzzy soft lattices. The organization of this paper is as follows: In Section 2, some basic concepts and results of the lattice theory, the soft set theory and the fuzzy lattices are introduced. In Section 3, the notion of fuzzy soft lattice is presented and some related properties are derived, then the lattice structure of fuzzy soft lattices is discussed. In Section 4 the notion of fuzzy soft ideal over a lattice is presented and some related properties are derived, then the lattice structures of fuzzy soft ideal over a lattice are discussed. Section 5 concludes the paper.

\section{Preliminaries}

In this section, we recall some basic concepts of the lattice theory, the soft set theory and the fuzzy lattices.

\subsection{Some relative concepts of lattice theory}

The general theory of partially ordered sets (posets) is based on a single undefined relationship. That of lattices is also based indirectly on this relationship, but directly on two dual binary operations which are analogous in many ways to ordinary addition and multiplication. It is this analogy which makes lattice theory a branch of algebra. By an upper bound to subset $X$ of a partially ordered set $P$ is meant an element $a \in P$ which is greater than every $x \in X$. A least upper bound is an upper bound lesser than every other upper bound of $x$. The notions of a lower bound and a greatest lower bound are defined dually. It is clear that a subset of a partially ordered set can have at most one l.u.b.(least upper bound) and one g.l.b. (greatest lower bound). ${ }^{24}$

Definition $1{ }^{24}$ A lattice is a partially ordered set $L$ such that any two of whose elements $x$ and $y$ have a g.l.b. or "meet" $x \wedge y$ and a l.u.b. or "join" $x \vee y$.

In other words, the lattice theory singles out a special type of poset for detailed investigation. However, we can also want such a characterization because if we can treat lattices as algebras, then all concepts and methods of universal algebra will become applicable. The usefulness of treating lattices as algebras will soon become clear.

Definition $2{ }^{21}$ An algebra $(L ; \wedge, \vee)$ is called a lattice if $L$ is a nonempty set, $\wedge$ and $\vee$ are binary operations on $L$, both $\wedge$ and $\vee$ are idempotent, commutative and associative, and they satisfy the two absorption identities, that is, for all $a, b, c \in L$ 
1. $a \wedge a=a, a \vee a=a$,

2. $a \wedge b=b \wedge a, a \vee b=b \vee a$,

3. $(a \wedge b) \wedge c=a \wedge(b \wedge c),(a \vee b) \vee c=a \vee(b \vee c)$,

4. $a \wedge(a \vee b)=a, a \vee(a \wedge b)=a$.

In fact, a lattice as an algebra and a lattice as a poset are "equivalent" concepts.

Throughout this paper, let $(L, \vee, \wedge)$ be a lattice(for short, $L$ ) unless otherwise specified.

A subset $L_{1}$ of $L$ is a sublattice of a lattice $L$ if and only if it satisfies: $x \wedge y \in L_{1}, x \vee y \in L_{1}$, for all $x \in L_{1}, y \in L_{1}$.

Definition $3{ }^{24,27} A$ homomorphism of a lattice $A$ into a lattice $B$ is a mapping $\varphi: A \rightarrow B$ such that $\varphi(x \wedge y)=\varphi(x) \wedge \varphi(y)$ and $\varphi(x \vee y)=\varphi(x) \vee \varphi(y)$ for all $x, y \in A$.

A non-empty subset $I$ of $L$ is called an ideal of $L$ if $x \vee y \in I$ and $\downarrow x \subseteq I$, for all $x, y \in I$. Dually, a non-empty subset $F$ of $L$ is called a filter of $L$ if $x \wedge y \in F$ and $\uparrow x \subseteq F$ ), for all $x, y \in F$, where, $\downarrow x=\{y \in L \mid y \leqslant x\}, \uparrow x=\{y \in L \mid y \geqslant x\} .{ }^{26}$

Theorem $1{ }^{24}$ Let $I_{i}$ be an ideal of $L$ for all $i \in K$, where $K$ is an index set. Then $\bigcap_{i \in K} I_{i}$ is an ideal of $L$.

Dually, we have the followings:

Theorem $2{ }^{24}$ Let $J_{i}$ be a filter of $L$ for all $i \in K$, where $K$ is an index set. Then $\bigcap_{i \in K} J_{i}$ is a filter of $L$.

Proposition $1{ }^{24}$ Let $\left(L_{1} ; \vee, \wedge\right),\left(L_{2} ; \vee, \wedge\right)$ be two sublattices of $L$, then, $\left(L_{1} \cap L_{2} ; \vee, \wedge\right)$ is a sublattice of $L$.

The following example shows that Proposition 1 doesn't hold in general with respect to the operation $\cup$.

Example 1 Let $L$ be a lattice as in Figure 1. It is clear that $L_{1}=\{0, a, d, 1\}$ and $L_{2}=\{0, a, c, 1\}$ are both sublattices of $L$. It is easily verified that $L_{1} \cap L_{2}=$ $\{0, a, 1\}$ is a sublattice and $L_{1} \cup L_{2}=\{0, a, c, d, 1\}$ isn't a sublattice of L because $c, d \in L_{1} \cup L_{2}$ and $c \wedge d=b \notin L_{1} \cup L_{2}$.

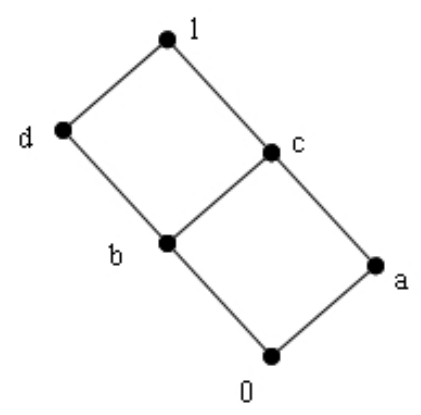

Fig. 1. Lattice $L$

Definition $4{ }^{24,21}$ A lattice $L$ is said to be modular if $M(a, b)$ holds for all $a, b \in L$, where, $M(a, b):(\forall x \in$ $L) x \leqslant b \Rightarrow x \vee(a \wedge b)=(x \vee a) \wedge b$.

Lemma $1{ }^{21}$ Consider the following two identities and inequality $(\forall x, y, z \in L)$ :

1. $(x \wedge y) \vee(x \wedge z)=x \wedge(y \vee z)$

2. $(x \vee y) \wedge(x \vee z)=x \vee(y \wedge z)$.

3. $(x \vee y) \wedge z \leqslant x \vee(y \wedge z)$.

Then (1)(2) and (3) are equivalent in any lattice $L$.

Remark $1{ }^{21,27}$ A lattice satisfying identities (1) or (2) is called distributive.

Note that (1) and (2) are not equivalent for fixed elements, that is, (1) can hold for three elements $a, b, c \in L$, whereas (2) does not.

\subsection{Some relative concepts of soft set theory}

In what follows, Let $U$ and $E$ be an initial universe set and a set of parameters, respectively. Let $P(U)$ denote the power set of $U$ and $A \subseteq E$ unless otherwise specified. Molodtsov defined the notion of a soft set in the following way,

A pair $(F, A)$ is called a soft set over $U$, where $F$ is a mapping given by $F: A \rightarrow P(U)$.

In other words, a soft set over $U$ is a parameterized family of subsets of the universe $U$. For $\epsilon \in A$, $F(\epsilon)$ may be considered as the set of $\epsilon$-approximate elements of the soft set $(F, A)$. 
Definition 5 9,5 The extended intersection of two soft sets $(F, A)$ and $(G, B)$ over a common universe $U$, denoted by $(F, A) \widetilde{\sqcap}(G, B)$, is the soft set $(H, C)$, where, $C=A \cup B$, and for all $e \in C$ :

$$
H(e)= \begin{cases}F(e), & \text { if } e \in A-B, \\ G(e), & \text { if } e \in B-A, \\ F(e) \cap G(e), & \text { if } e \in A \cap B .\end{cases}
$$

Definition $6{ }^{4,5}$ The union of two soft sets $(F, A)$ and $(G, B)$ over a common universe $U$, denoted by $(F, A) \widetilde{\sqcup}(G, B)$, is the soft set $(H, C)$, where $C=A \cup B$, and $\forall e \in C$, if $e \in A-B$, then $H(e)=F(e)$; if $e \in B-A$ then $H(e)=G(e)$; if $e \in A \cap B$, then $H(e)=F(e) \cup$ $G(e)$, that is, for all $e \in C$ :

$$
H(e)= \begin{cases}F(e), & \text { if } e \in A-B, \\ G(e), & \text { if } e \in B-A, \\ F(e) \cup G(e), & \text { if } e \in A \cap B .\end{cases}
$$

Definition $7^{9,5}$ The restricted intersection of two soft sets $(F, A)$ and $(G, B)$ over a common universe $U$, denoted by $(F, A) \cap(G, B)$, is the soft set $(H, C)$, where $C=A \cap B$, and $\forall e \in C, H(e)=F(e) \cap G(e)$.

Definition $8{ }^{9,5}$ The restricted union of two soft sets $(F, A)$ and $(G, B)$ over a common universe $U$, denoted by $(F, A) \cup(G, B)$, is the soft set $(H, C)$, where $C=A \cap B$, and $\forall e \in C, H(e)=F(e) \cup G(e)$.

Definition $9{ }^{9,5}(F, A)$ is called a relative null soft set (with respect to the parameter set $A$ ), denoted by $\emptyset_{A}$, if $F(A)=\varnothing$ for all $e \in A$.

$(F, A)$ is called a relative whole soft set (with respect to the parameter set $A$ ), denoted by $U_{A}$, if $F(e)=U$ for all $e \in A$.

\subsection{Some relative concepts of fuzzy lattice}

Definition $10{ }^{13,2}$ Let $U$ be an initial universal set and let $E$ be a set of parameters. Let $I^{U}$ denote the set of all fuzzy subsets of $U, A \subseteq E, A$ pair $(F, A)$ is called a fuzzy soft set over $U$, where, $F$ is a mapping given by $F: A \rightarrow I^{U}$.

Definition $11{ }^{26}$ A fuzzy subset of $L$ is a function $\mu: L \rightarrow[0,1]$. A fuzzy subset $\mu$ of $L$ is called a fuzzy sublattice of $L$ if $\mu(x \wedge y) \wedge \mu(x \vee y) \geqslant \mu(x) \wedge \mu(y)$ for all $x, y \in L$. Let $\mu$ be a fuzzy sublattice of $L$. Then
1. $\mu$ is a fuzzy ideal of $L$, if $\mu(x \vee y)=\mu(x) \wedge \mu(y)$, for all $x, y \in L$.

2. $\mu$ is a fuzzy filter of $L$, if $\mu(x \wedge y)=\mu(x) \wedge \mu(y)$, for all $x, y \in L$.

Let us illustrate this definition using the following example.

Example 2 Let $L=\{0, a, b, c, d, 1\}$ and define $\wedge$ and $\checkmark$ by the following Cayley tables:

Table 1. the definition of the operator $\wedge$ on $L$

\begin{tabular}{c|cccccc}
\hline$\wedge$ & 0 & $a$ & $b$ & $c$ & $d$ & 1 \\
\hline 0 & 0 & 0 & 0 & 0 & 0 & 0 \\
$a$ & 0 & $a$ & $a$ & $a$ & $a$ & $a$ \\
$b$ & 0 & $a$ & $b$ & $a$ & $b$ & $b$ \\
$c$ & 0 & $a$ & $a$ & $c$ & $c$ & $c$ \\
$d$ & 0 & $a$ & $b$ & $c$ & $d$ & $d$ \\
1 & 0 & $a$ & $b$ & $c$ & $d$ & 1 \\
\hline
\end{tabular}

Table 2. the definition of the operator $\vee$ on $L$

\begin{tabular}{c|cccccc}
\hline$\vee$ & 0 & $a$ & $b$ & $c$ & $d$ & 1 \\
\hline 0 & 0 & $a$ & $b$ & $c$ & $d$ & 1 \\
$a$ & $a$ & $a$ & $b$ & $c$ & $d$ & 1 \\
$b$ & $b$ & $b$ & $b$ & $d$ & $d$ & 1 \\
$c$ & $c$ & $c$ & $d$ & $c$ & $d$ & 1 \\
$d$ & $d$ & $d$ & $d$ & $d$ & $d$ & 1 \\
1 & 1 & 1 & 1 & 1 & 1 & 1 \\
\hline
\end{tabular}
$\{0 / 0.3, a / 0.8, b / 0.6, c / 0.4, d / 0.4,1 / 0\}$, then routine verification reveals that $\mu$ is a fuzzy sublattice of $L$.

Proposition $2{ }^{25,26}$ Let $\mu$ be a fuzzy sublattice of $L$. Then

1. $\mu$ is a fuzzy ideal of $L$ if and only if $x \leqslant y$ implies that $\mu(x) \geqslant \mu(y)$, for all $x, y \in L$.

2. $\mu$ is a fuzzy filter of $L$ if and only if $x \leqslant y$ implies that $\mu(x) \leqslant \mu(y)$, for all $x, y \in L$.

It is clear that if $\alpha_{1} \geqslant \alpha_{2}$, then $\mu_{\alpha_{1}} \subseteq \mu_{\alpha_{2}} . \mu$ is a fuzzy sublattice if and only if $\mu_{\alpha}$ is a sublattice of $L$ for all $\alpha \in[0,1]$, where, $\mu_{\alpha}=\{x \in L \mid \mu(x) \geqslant \alpha\}$.

By means of the above concepts and operations of soft sets, we can propose the concepts of fuzzy soft lattices and study some related properties. 


\section{Fuzzy soft lattices and the lattice structure of fuzzy soft lattices}

Algebraic structures play a fundamental role in many fields of mathematics. In this section, we discuss the lattice structure of fuzzy soft lattices. For this, we present the notions of fuzzy soft lattices and discuss the properties of lattices and fuzzy lattices at first.

Definition 12 Let $S \subseteq L$. If there exists a sublattice of $L$, denoted by $\mathscr{L}(S)$, such that if $S \subseteq S^{\prime}$, then $\mathscr{L}(S) \subseteq S^{\prime}$, where, $S^{\prime}$ is an arbitrary sublattice of $L$, then the sublattice $\mathscr{L}(S)$ is called the sublattice generated by $S$.

Lemma 2 Let $S \subseteq L$, then $\mathscr{L}(S)$ must be exist.

Proof. Let

$\widetilde{S}=\left\{S_{1} \mid S_{1}\right.$ is a sublattice containing $S$ of $\left.L\right\}$. We prove that $\mathscr{L}(S)=\bigcap_{S_{i} \in \widetilde{S}} S_{i}$.

1. It is clear that $\cap S_{i}$ is a sublattice of $L$. $S_{i} \in \widetilde{S}$

2. If $S^{\prime}$ is an arbitrary sublattice containing $S$ of $L$, then $S^{\prime} \in \widetilde{S}$, so we have $\bigcap_{\widetilde{S}} S_{i} \subseteq S^{\prime}$. By Definition 12, we can get that $\cap S_{i}$ is the sublattice generated by $S$.

Corollary 1 Let $S \subseteq L$, then $\mathscr{L}(S)$ must be unique.

Lemma 3 1. If $S$ is a subset of $L$, then $S \subseteq$ $\mathscr{L}(S)$.

2. If $S$ is a subset of $L$, then $S=\mathscr{L}(S)$ iff $S$ is a sublattice of $L$.

3. If $S_{1} \subseteq S_{2}$, then $\mathscr{L}\left(S_{1}\right) \subseteq \mathscr{L}\left(S_{2}\right)$.

4. If $L_{1}$ is a sublattice of $L, S_{1} \subseteq L_{1}, S_{2} \subseteq L_{1}$, then $\mathscr{L}\left(S_{1} \cup S_{2}\right) \subseteq L_{1}$.

5. Let $S_{1}, S_{2}$ be subsets of $L$, then $\mathscr{L}\left(S_{1} \cup S_{2}\right)=$ $\mathscr{L}\left(\mathscr{L}\left(S_{1}\right) \cup \mathscr{L}\left(S_{2}\right)\right)$.

Proof.

(1)(2)(3)(4) are trivial. We only prove (5).

(5) (i)Since $S_{1} \subseteq \mathscr{L}\left(S_{1}\right), S_{2} \subseteq \mathscr{L}\left(S_{2}\right)$, we can get that $S_{1} \cup S_{2} \subseteq \mathscr{L}\left(S_{1}\right) \cup \mathscr{L}\left(S_{2}\right)$ and then $\mathscr{L}\left(S_{1} \cup\right.$ $\left.S_{2}\right) \subseteq \mathscr{L}\left(\mathscr{L}\left(S_{1}\right) \cup \mathscr{L}\left(S_{2}\right)\right)$.

(ii) Let $\mathscr{L}\left(S_{1}\right)=\bigcap_{S_{1 i} \in \widetilde{S}_{1}} S_{1 i}, \mathscr{L}\left(S_{2}\right)=\bigcap_{S_{2 i} \in \widetilde{S}_{2}} S_{2 i}$,

where $\widetilde{S}_{1}=\left\{S_{1 i} \mid S_{1 i}\right.$ is a sublattice containing $S_{1}$ of $\left.L\right\}$, $\widetilde{S}_{2}=\left\{S_{2 i} \mid S_{2 i}\right.$ is a sublattice containing $S_{2}$ of $\left.L\right\}$.

$\mathscr{L}\left(\mathscr{L}\left(S_{1}\right) \cup \mathscr{L}\left(S_{2}\right)\right)=\mathscr{L}\left(\bigcap_{S_{1 i} \in \widetilde{S}_{1}} S_{1 i} \cup \bigcap_{S_{2 j} \in \widetilde{S}_{2}} S_{2 j}\right)=$

$\mathscr{L}\left(\cap \cap\left(S_{1 i} \cup S_{2 j}\right)\right) \subseteq \mathscr{L}\left(S_{1} \cup S_{2}\right)$.

$S_{1 i} \in \widetilde{S}_{1} S_{2 j} \in \widetilde{S}_{2}$

Let $L$ be a lattice defined in Figure 1 in Example 1. Suppose that $A=\{0, a, b\}, B=\{b, c, d, 1\}$, then routine verification reveals that (5) doesn't hold in general with respective to $\cap$, that is, $\mathscr{L}\left(S_{1} \cap S_{2}\right) \neq$ $\mathscr{L}\left(\mathscr{L}\left(S_{1}\right) \cap \mathscr{L}\left(S_{2}\right)\right)$, that is, $\mathscr{L}\left(S_{1} \cap S_{2}\right) \neq \mathscr{L}\left(S_{1}\right) \cap$ $\mathscr{L}\left(S_{2}\right)$.

Definition 13 Let $L_{1}, L_{2}$ be two sublattices of $L$, the operator $\sqcap$ is defined by $L_{1} \sqcap L_{2}=L_{1} \cap L_{2}$; the operator $\sqcup$ is defined by $L_{1} \sqcup L_{2}=\mathscr{L}\left(L_{1} \cup L_{2}\right)$.

Lemma 4 Let $L_{1}, L_{2}$ and $L_{3}$ be sublattices of $L$. Then

1. $L_{1} \sqcap L_{1}=L_{1}$,

2. $L_{1} \sqcap L_{2}=L_{2} \sqcap L_{1}$,

3. $\left(L_{1} \sqcap L_{2}\right) \sqcap L_{3}=L_{1} \sqcap\left(L_{2} \sqcap L_{3}\right)$.

4. $\left(L_{1} \sqcap L_{2}\right) \sqcup L_{1}=L_{1}$.

Proof. Straightforward.

Lemma 5 Let $L_{1}, L_{2}$ and $L_{3}$ be sublattices of $L$. Then

1. $L_{1} \sqcup L_{1}=L_{1}$,

2. $L_{1} \sqcup L_{2}=L_{2} \sqcup L_{1}$,

3. $\left(L_{1} \sqcup L_{2}\right) \sqcup L_{3}=L_{1} \sqcup\left(L_{2} \sqcup L_{3}\right)$.

4. $\left(L_{1} \sqcup L_{2}\right) \sqcap L_{1}=L_{1}$.

Proof. Straightforward.

Theorem $3\left(2^{L}, \sqcup, \sqcap\right)$ forms a lattice, where, $2^{L}$ denotes the set of all sublattices of $L$, i.e., $2^{L}=$ $\left\{L_{1} \mid L_{1}\right.$ is a sublattice of $\left.L\right\}$.

Proof. It is immediate by Lemma 4 and Lemma 5 . 
Example 3 Let $L$ be a defined in Figure 1 in Example 1. Suppose that $L_{1}=\{a, c\}, L_{2}=\{0, d\}, L_{3}=$ $\{0, a, b, c\}$, routine verification reveals that $L_{1}, L_{2}$ and $L_{3}$ are sublattices of $L$ and $L_{1} \subseteq L_{3}$, but $\left(L_{1} \sqcup\right.$ $\left.L_{2}\right) \sqcap L_{3}=L_{3}, L_{1} \sqcup\left(L_{2} \sqcap L_{3}\right)=\{a, c, 0\}$, so, $\left(2^{L}, \sqcup, \sqcap\right)$ doesn't form a modular lattice in general.

Definition 14 Let $\mu_{1}, \mu_{2}$ be two fuzzy sublattices of $L$, their intersection, denoted by $\mu_{1} \sqcap \mu_{2}$, is defined by $\left(\mu_{1} \sqcap \mu_{2}\right)(x)=\mu_{1}(x) \wedge \mu_{2}(x)$, for all $x \in L$.

Lemma 6 Let $\mu_{1}, \mu_{2}$ be two fuzzy sublattices of $L$, then their intersection $\mu_{1} \sqcap \mu_{2}$ is a fuzzy sublattice of $L$.

Proof. For all $x \in L, y \in L$, by Definition 14, we have that

$\left(\mu_{1} \sqcap \mu_{2}\right)(x \wedge y) \wedge\left(\mu_{1} \sqcap \mu_{2}\right)(x \vee y)$

$=\mu_{1}(x \wedge y) \wedge \mu_{2}(x \wedge y) \wedge \mu_{1}(x \vee y) \wedge \mu_{2}(x \vee y)$

$\geqslant \mu_{1}(x) \wedge \mu_{1}(y) \wedge \mu_{2}(x) \wedge \mu_{2}(y)$

$=\left(\mu_{1} \sqcap \mu_{2}\right)(x) \wedge\left(\mu_{1} \sqcap \mu_{2}\right)(y)$.

By Definition 8, we can get that $\mu_{1} \sqcap \mu_{2}$ is a fuzzy sublattice of $L$.

Lemma 7 Let $\mu_{1}, \mu_{2}$ and $\mu_{3}$ be fuzzy sublattices of L. Then

$$
\begin{aligned}
& \text { 1. } \mu_{1} \sqcap \mu_{1}=\mu_{1}, \\
& \text { 2. } \mu_{1} \sqcap \mu_{2}=\mu_{2} \sqcap \mu_{1}, \\
& \text { 3. }\left(\mu_{1} \sqcap \mu_{2}\right) \sqcap \mu_{3}=\mu_{1} \sqcap\left(\mu_{2} \sqcap \mu_{3}\right) .
\end{aligned}
$$

Proof. It is immediate by Definition 14 and Lemma 6.

Lemma 8 Let $\mu_{1}$ be a fuzzy subset of $L$, then $\mu$ is the least fuzzy sublattice of $L$ containing $\mu_{1}$, that is, if $\mu^{\prime}$ is an arbitrary fuzzy sublattice of $L$ and $\mu_{1} \subseteq \mu$, then $\mu \subseteq \mu^{\prime}$. Where, $\mu$ is defined by $\mu(x)=\inf S(\mu(x))=$ $\inf \left\{\mu(x) \mid \mu(x \wedge y) \wedge \mu(x \vee y) \geqslant \mu(x) \wedge \mu(y) \geqslant \mu_{1}(x) \vee\right.$ $\left.\mu_{1}(y), \forall y \in L\right\} . \mu$ is called the fuzzy sublattice of $L$ generated by $\mu_{1}$, denoted by $\mathscr{L}\left(\mu_{1}\right)$.

Proof. It is clear that $\mu$ is a fuzzy sublattice of $L$ containing $\mu_{1}$, so we only prove that an arbitrary fuzzy sublattice $\mu^{\prime}$ of $L$ containing $\mu_{1}$ must contain $\mu$. For all $x \in L$, since $\mu^{\prime}(x) \geqslant \mu_{1}(x)$ and $\mu^{\prime}(x \wedge y) \wedge \mu^{\prime}(x \vee y) \geqslant \mu^{\prime}(x) \wedge \mu^{\prime}(y)$, so $\mu^{\prime}(x) \subseteq S(\mu)$, so $\mu^{\prime}(x) \geqslant \mu(x)$, thus, $\mu \subseteq \mu^{\prime}$, that is, $\mu$ is the least fuzzy sublattice of $L$.
Corollary $2 \mu=\mathscr{L}\left(\mu_{1} \cup \mu_{2}\right)$ is the least fuzzy lattice containing $\mu_{1}$ and $\mu_{2}$, where, $\left(\mu_{1} \cup \mu_{2}\right)(x)=$ $\max \left\{\mu_{1}(x), \mu_{2}(x)\right\}$.

Proof. It is immediate by Lemma 8 .

Based on Lemma 8 and Corollary $2, \mu=\mathscr{L}\left(\mu_{1} \cup \mu_{2}\right)$ can be called the fuzzy sublattice of $L$ generated by $\mu_{1}, \mu_{2}$, denoted by $\mu_{1} \sqcup \mu_{2}$.

Lemma 9 1. If $\mu$ is a fuzzy subset of $L$, then $\mu \subseteq \mathscr{L}(\mu)$,

2. If $\mu$ is a subset of $L$, then $\mu=\mathscr{L}(\mu)$ iff $\mu$ is a fuzzy sublattice of $L$.

3. If $\mu_{1} \subseteq \mu_{2}$, then $\mathscr{L}\left(\mu_{1}\right) \subseteq \mathscr{L}\left(\mu_{2}\right)$.

4. If $\mu_{1}, \mu_{2}$ are fuzzy subsets of $L, \mu_{1} \subseteq \mu, \mu_{2} \subseteq \mu$, and $\mu$ is a fuzzy sublattice of $L$, then $\mathscr{L}\left(\mu_{1} \cup\right.$ $\left.\mu_{2}\right) \subseteq \mu$.

Proof. Straightforward.

Lemma 10 Let $\mu_{1}, \mu_{2}$ be fuzzy subsets of $L$, then $\mathscr{L}\left(\mu_{1}\right) \sqcup \mathscr{L}\left(\mu_{2}\right)=\mathscr{L}\left(\mu_{1} \cup \mu_{2}\right)$.

Proof.

1. Since $\mu_{1} \subseteq \mu_{1} \cup \mu_{2}$, we can get that $\mathscr{L}\left(\mu_{1}\right) \subseteq$ $\mathscr{L}\left(\mu_{1} \cup \mu_{2}\right)$. Similarly, we have that $\mathscr{L}\left(\mu_{2}\right) \subseteq$ $\mathscr{L}\left(\mu_{1} \cup \mu_{2}\right)$. So, by Lemma 9, $\mathscr{L}\left(\mu_{1}\right) \sqcup$ $\mathscr{L}\left(\mu_{2}\right) \subseteq \mathscr{L}\left(\mu_{1} \cup \mu_{2}\right)$.

2. Since $\mu_{1} \subseteq \mathscr{L}\left(\mu_{1}\right), \mu_{2} \subseteq \mathscr{L}\left(\mu_{2}\right)$, so $\mu_{1} \cup \mu_{2} \subseteq$ $\mathscr{L}\left(\mu_{1}\right) \cup \mathscr{L}\left(\mu_{2}\right)$, moreover, $\mathscr{L}\left(\mu_{1} \cup \mu_{2}\right) \subseteq$ $\mathscr{L}\left(\mathscr{L}\left(\mu_{1}\right) \cup \mathscr{L}\left(\mu_{2}\right)\right)$, that is, $\mathscr{L}\left(\mu_{1} \cup \mu_{2}\right) \subseteq$ $\mathscr{L}\left(\mu_{1}\right) \sqcup \mathscr{L}\left(\mu_{2}\right)$.

Sum up above, we can get the result as required.

Lemma 11 Let $\mu_{1}, \mu_{2}$ and $\mu_{3}$ be fuzzy sublattices of L. Then

1. $\mu_{1} \sqcup \mu_{1}=\mu_{1}$,

2. $\mu_{1} \sqcup \mu_{2}=\mu_{2} \sqcup \mu_{1}$,

3. $\left(\mu_{1} \sqcup \mu_{2}\right) \sqcup \mu_{3}=\mu_{1} \sqcup\left(\mu_{2} \sqcup \mu_{3}\right)$,

Proof. It is immediate by Lemma 8 .

Lemma 12 Let $\mu_{1}$ and $\mu_{2}$ be fuzzy sublattices of $L$. Then

1. $\left(\mu_{1} \sqcup \mu_{2}\right) \sqcap \mu_{1}=\mu_{1}$,

2. $\left(\mu_{1} \sqcap \mu_{2}\right) \sqcup \mu_{1}=\mu_{1}$. 
Proof. We only prove (1).

For all $x \in L$, since $\left(\mu_{1} \sqcup \mu_{2}\right)(x)=\inf \{\mu(x) \mid \mu(x \wedge$ $\left.y) \wedge \mu(x \vee y) \geqslant \mu(x) \wedge \mu(y) \geqslant \mu_{1}(x) \vee \mu_{2}(y)\right\} \geqslant$ inf $\left\{\mu(x) \mid \mu(x) \geqslant \mu_{1}(x)\right\}=\mu_{1}(x)$, so we can get that $\left(\mu_{1} \sqcup \mu_{2}\right)(x) \wedge \mu_{1}(x)=\mu_{1}(x)$, that is, $\left(\mu_{1} \sqcup \mu_{2}\right) \sqcap \mu_{1}=$ $\mu_{1}$.

Lemma 13 Let $\mu_{1}, \mu_{2}$ and $\mu_{3}$ be fuzzy sublattices of L. Then,

1. $\left(\mu_{1} \sqcup \mu_{2}\right) \sqcap \mu_{3}=\left(\mu_{1} \sqcap \mu_{3}\right) \sqcup\left(\mu_{2} \sqcap \mu_{3}\right)$.

2. $\left(\mu_{1} \sqcap \mu_{2}\right) \sqcup \mu_{3}=\left(\mu_{1} \sqcup \mu_{3}\right) \sqcap\left(\mu_{2} \sqcup \mu_{3}\right)$.

Proof. (1)We only prove $\left(\mu_{1} \sqcup \mu_{2}\right) \sqcap \mu_{3} \leqslant\left(\mu_{1} \sqcap \mu_{3}\right) \sqcup$ $\left(\mu_{2} \sqcap \mu_{3}\right)$.

For all $x \in L$, without loss of generality, we suppose $\mu_{1}(x) \leqslant \mu_{2}(x)$.

(i) If $\mu_{3}(x) \geqslant \mu_{2}(x) \geqslant \mu_{1}(x)$, then

$\left(\left(\mu_{1} \sqcap \mu_{3}\right) \sqcup\left(\mu_{2} \sqcap \mu_{3}\right)\right)(x)=\left(\mu_{1}(x) \sqcap \mu_{3}(x)\right) \sqcup\left(\mu_{2}(x) \sqcap\right.$ $\left.\mu_{3}(x)\right)$

$=\mu_{1}(x) \sqcup \mu_{2}(x) \geqslant\left(\left(\mu_{1} \sqcup \mu_{2}\right) \sqcap \mu_{3}\right)(x) ;$

(ii)If $\mu_{2}(x) \geqslant \mu_{3}(x) \geqslant \mu_{1}(x)$, then

$\left(\left(\mu_{1} \sqcap \mu_{3}\right) \sqcup\left(\mu_{2} \sqcap \mu_{3}\right)\right)(x)=\left(\mu_{1}(x) \sqcap \mu_{3}(x)\right) \sqcup\left(\mu_{2}(x) \sqcap\right.$ $\left.\mu_{3}(x)\right)$

$=\mu_{1}(x) \sqcup \mu_{3}(x) \geqslant\left(\left(\mu_{1} \sqcup \mu_{2}\right) \sqcap \mu_{3}\right)(x) ;$

(iii) If $\mu_{2}(x) \geqslant \mu_{1}(x) \geqslant \mu_{3}(x)$, then

$\left(\left(\mu_{1} \sqcap \mu_{3}\right) \sqcup\left(\mu_{2} \sqcap \mu_{3}\right)\right)(x)=\left(\mu_{1}(x) \sqcap \mu_{3}(x)\right) \sqcup\left(\mu_{2}(x) \sqcap\right.$

$\left.\mu_{3}(x)\right)=$

$\mu_{3}(x) \sqcup \mu_{3}(x) \geqslant\left(\left(\mu_{1} \sqcup \mu_{2}\right) \sqcap \mu_{3}\right)(x) ;$

Therefore, we conclude that $\left(\mu_{1} \sqcup \mu_{2}\right) \sqcap \mu_{3}=\left(\mu_{1} \sqcap\right.$ $\left.\mu_{3}\right) \sqcup\left(\mu_{2} \sqcap \mu_{3}\right)$.

(2) For all $x \in L$, without loss of generality, we suppose $\mu_{1}(x) \leqslant \mu_{2}(x)$.

(i) If $\mu_{3}(x) \geqslant \mu_{2}(x) \geqslant \mu_{1}(x)$, then

$\left(\left(\mu_{1} \sqcup \mu_{3}\right) \sqcap\left(\mu_{2} \sqcup \mu_{3}\right)\right)(x)=\left(\mu_{1} \sqcup \mu_{3}\right)(x) \wedge\left(\mu_{2} \sqcup \mu_{3}\right)(x)$

$=\mu_{3}(x)=\left(\left(\mu_{1} \sqcap \mu_{2}\right) \sqcup \mu_{3}\right)(x)$;

(ii)If $\mu_{2}(x) \geqslant \mu_{3}(x) \geqslant \mu_{1}(x)$, then

$\left(\left(\mu_{1} \sqcup \mu_{3}\right) \sqcap\left(\mu_{2} \sqcup \mu_{3}\right)\right)(x)=\left(\mu_{1} \sqcup \mu_{3}\right)(x) \sqcap\left(\mu_{2} \sqcup \mu_{3}\right)(x)$

$=\mu_{3}(x)=\left(\left(\mu_{1} \sqcap \mu_{2}\right) \sqcup \mu_{3}\right)(x)$;

(iii) If $\mu_{2}(x) \geqslant \mu_{1}(x) \geqslant \mu_{3}(x)$, then

$\left(\left(\mu_{1} \sqcup \mu_{3}\right) \sqcap\left(\mu_{2} \sqcup \mu_{3}\right)\right)(x)=\left(\mu_{1} \sqcup \mu_{3}\right)(x) \sqcap\left(\mu_{2}(x) \sqcup\right.$ $\left.\mu_{3}\right)(x)$

$\left.=\mu_{1}(x) \sqcap \mu_{2}(x)=\left(\left(\mu_{1} \sqcap \mu_{2}\right) \sqcup \mu_{3}\right)\right)(x) ;$

Therefore, we conclude that $\left(\mu_{1} \sqcup \mu_{2}\right) \sqcap \mu_{3}=\left(\mu_{1} \sqcap\right.$ $\left.\mu_{3}\right) \sqcup\left(\mu_{2} \sqcap \mu_{3}\right)$.
Theorem 4 Let $\mathscr{F}(L)$ denotes the set of all fuzzy sublattices of $L$. Then $(\mathscr{F}(L) ; \sqcup, \square)$ forms a distributive lattice.

Proof. It is immediate by the proof of Lemma 7, Lemma 11, Lemma 12 and Lemma 13.

Definition 15 Let $(F, A)$ be a fuzzy soft set over $L$, $(F, A)$ is called a fuzzy soft lattice if $F(\varepsilon)$ is a fuzzy sublattice of $L$ for each $\varepsilon \in A$.

Definition 16 Let $(F, A)$ and $(E, B)$ be fuzzy soft lattices over $L$. We define $(F, A) \widetilde{\sqcup}(E, B)=(H, C)$ as follows: $C=A \cup B$ and for all $e \in C$

$$
H(e)= \begin{cases}F(e), & \text { if } e \in A-B, \\ E(e), & \text { if } e \in B-A, \\ F(e) \sqcup G(e), & \text { if } e \in A \cap B .\end{cases}
$$

Proposition 3 Let $(F, A)$ and $(E, B)$ be fuzzy soft lattices over $L$. Then $(H, C)=(F, A) \widetilde{\sqcup}(E, B)$ is a fuzzy soft lattice over $L$.

Proof. It is immediate by Definition 16 .

Proposition 4 Let $(F, A),(G, B)$ and $(H, C)$ be fuzzy soft lattices over $L$. Then

1. $(F, A) \widetilde{\sqcup}(F, A)=(F, A)$,

2. $(F, A) \widetilde{\sqcup}(G, B)=(G, B) \widetilde{\sqcup}(F, A)$,

3. $((F, A) \widetilde{\sqcup}(G, B)) \widetilde{\sqcup}(H, C)=(F, A) \widetilde{\square}((G, B) \widetilde{\sqcup}(H, C))$.

Proof. (1) and (2) are trivial. We only prove (3). Suppose that $((F, A) \widetilde{\sqcup}(G, B)) \widetilde{\sqcup}(H, C)=(K, A \cup B \quad C \quad C)$, $(F, A) \widetilde{\sqcup}((G, B) \widetilde{\sqcup}(H, C))=(M, A \cup B \cup C))$.

For all $\varepsilon \in A \cup B \cup C$, it follows that $\varepsilon \in A$ or $\varepsilon \in B$ or $\varepsilon \in C$. Without loss of generality, we can suppose that $\varepsilon \in C$,

(a) If $\varepsilon \notin A$ and $\varepsilon \notin B$, then $K(\varepsilon)=H(\varepsilon)=M(\varepsilon)$.

(b) If $\varepsilon \in A$ and $\varepsilon \notin B$, then $K(\varepsilon)=F(\varepsilon) \sqcup H(\varepsilon)=$ $M(\varepsilon)$.

(c) If $\varepsilon \notin A$ and $\varepsilon \in B$, then $K(\varepsilon)=G(\varepsilon) \sqcup H(\varepsilon)=$ $M(\varepsilon)$.

(d) If $\varepsilon \in A$ and $\varepsilon \in B$, then $K(\varepsilon)=(F(\varepsilon) \sqcup G(\varepsilon)) \sqcup$ $H(\varepsilon)=F(\varepsilon) \sqcup(G(\varepsilon) \sqcup H(\varepsilon))=M(\varepsilon)$.

Since $K$ and $M$ are indeed the same set-valued mappings, we conclude that 
$((F, A) \widetilde{\sqcup}(G, B)) \widetilde{\sqcup}(H, C)=(F, A) \widetilde{\sqcup}((G, B) \widetilde{\sqcup}(H, C))$ as required.

Similarly, we have the followings:

Definition 17 Let $(F, A)$ and $(G, B)$ be fuzzy soft lattices over $L$. We define $(F, A) \widetilde{\sqcap}(G, B)=(H, C)$ as follows:

$$
H(\varepsilon)=\left\{\begin{array}{lll}
F(\varepsilon), & \text { if } \quad \varepsilon \in A-B, \\
G(\varepsilon), & \text { if } \quad \varepsilon \in B-A, \\
F(\varepsilon) \sqcap G(\varepsilon), & \text { if } \quad \varepsilon \in A \cap B .
\end{array}\right.
$$

Proposition 5 If $(F, A)$ and $(G, B)$ be fuzzy soft lattices over $L$, then $(H, C)=(F, A) \widetilde{\sqcap}(G, B)$ is a fuzzy soft lattice over $L$.

Proof. Straightforward.

Proposition 6 Let $(F, A),(G, B)$ and $(H, C)$ be fuzzy soft lattices over $L$. Then

1. $(F, A) \widetilde{\sqcap}(F, A)=(F, A)$,

2. $(F, A) \widetilde{\sqcap}(G, B)=(G, B) \widetilde{\Pi}(F, A)$,

3. $((F, A) \widetilde{\sqcap}(G, B)) \widetilde{\sqcap}(H, C)=(F, A) \widetilde{\sqcap}((G, B) \widetilde{\Pi}(H, C))$.

Proof. It is similar to the proof of Proposition 4 .

Proposition 7 Let $(F, A),(G, B)$ be fuzzy soft lattices over L. Then

1. $((F, A) \widetilde{\sqcup}(G, B)) \widetilde{\Pi}(F, A)=(F, A)$,

2. $((F, A) \widetilde{\sqcap}(G, B)) \widetilde{\sqcup}(F, A)=(F, A)$.

\section{Proof.}

1. Suppose that $(F, A) \widetilde{\sqcup}(G, B)=(H, A \cup B)$ and $((F, A) \widetilde{\sqcup}(G, B)) \widetilde{\sqcap}(F, A)=(K,(A \cup B) \cup A)=$ $(K, A)$. For all $\varepsilon \in A$,

(a) if $\varepsilon \in B$, then $\varepsilon \in A \cup B$, so $K(\varepsilon)=H(\varepsilon) \cap$ $F(\varepsilon)=(F(\varepsilon) \sqcup G(\varepsilon)) \cap F(\varepsilon)=F(\varepsilon)$;

(b)if $\varepsilon \notin B$, then $\varepsilon \in A \cup B$, so $K(\varepsilon)=H(\varepsilon) \sqcap$

$F(\varepsilon)=F(\varepsilon) \sqcap F(\varepsilon)=F(\varepsilon)$.

Hence $((F, A) \widetilde{\sqcup}(G, B)) \widetilde{\Pi}(F, A)=(F, A)$.

2. Suppose that $(F, A) \widetilde{\sqcap}(G, B)=(H, A \cup B)$ and $((F, A) \widetilde{\sqcap}(G, B)) \widetilde{\sqcup}(F, A)=(K,(A \cup B) \cup A)=$ $(K, A)$. For all $\varepsilon \in A$,

(a) if $\varepsilon \in B$, then $\varepsilon \in A \cup B$, so we have that
$K(\varepsilon)=H(\varepsilon) \sqcup F(\varepsilon)=(F(\varepsilon) \sqcap G(\varepsilon)) \sqcup F(\varepsilon)=$ $F(\varepsilon)$;

(b)if $\varepsilon \notin B$, then, since $\varepsilon \in A$, so $\varepsilon \in A \cup B$ and $\varepsilon \notin A \cap B$, thus we have that $K(\varepsilon)=H(\varepsilon) \sqcup$ $F(\varepsilon)=F(\varepsilon) \sqcup F(\varepsilon)=F(\varepsilon)$.

Hence $((F, A) \widetilde{\sqcap}(G, B)) \widetilde{\sqcup}(F, A)=(F, A)$.

\section{Definition 18 Let}

$S(L, E)=\{(F, A) \mid A \subseteq E$, and $(F, A)$ is a fuzzy soft lattice over $L\}$,

then $S(L, E)$ is called a fuzzy soft class.

Definition 19 Suppose that $S\left(L_{1}, E\right)$ and $S\left(L_{2}, E^{\prime}\right)$ are fuzzy soft classes. Let $\mu: L_{1} \rightarrow L_{2}$ and $p$ : $E \rightarrow E^{\prime}$ be mappings. If $(\Lambda, \Sigma) \in S\left(L_{1}, E\right)$, the image of $(\Lambda, \Sigma)$ under the function $f=(\mu, p)$, denoted by $f(\Lambda, \Sigma)$, is the fuzzy soft set over $L_{2}$ defined by $f(\Lambda, \Sigma)=(\mu(\Lambda), p(\Sigma))$ as the followings, where, for all $\beta \in p(\Sigma), y \in L_{2}$.

$\mu(\Lambda)(\beta)(y)= \begin{cases}\bigvee_{x \in \mu^{-1}(y)} \bigvee_{\alpha \in p^{-1}(\beta) \cap \Sigma} \Lambda(\alpha)(x), & \text { if } \mu^{-1}(y) \neq \phi, \\ 0, & \text { otherwise. }\end{cases}$

Definition 20 Let $S\left(L_{1}, E\right)$ and $S\left(L_{2}, E^{\prime}\right)$ be fuzzy soft classes. Let $\mu: L_{1} \rightarrow L_{2}$ and $p: E \rightarrow E^{\prime}$ be mappings, $(\Delta, \Omega) \in S\left(L_{2}, E^{\prime}\right)$. The inverse image of $(\Delta, \Omega)$ under the function $f=(\mu, p)$, denoted by $f^{-1}(\Delta, \Omega)$, is the fuzzy soft set over $L_{1}$ defined by $f^{-1}(\Delta, \Omega)=\left(\mu^{-1}(\Delta), p^{-1}(\Omega)\right)$, where $\mu^{-1}(\Delta)(\alpha)(x)=$ $\Delta(p(\alpha))(\mu(x)), \forall \alpha \in p^{-1}(\Omega), \forall x \in L_{1}$.

Theorem 5 Let $S\left(L_{1}, E\right)$ and $S\left(L_{2}, E^{\prime}\right)$ be fuzzy soft classes, $p: E \rightarrow E^{\prime}$ be a mapping and $\mu$ be a homomorphic mapping from $L_{1}$ to $L_{2}$. If $(\Delta, \Omega) \in$ $S\left(L_{2}, E^{\prime}\right)$, then $f^{-1}(\Delta, \Omega)$ is a fuzzy soft lattice over $L_{1}$.

Proof. For all $\varepsilon \in p^{-1}(\Omega), x_{1}, x_{2} \in L$, $\mu^{-1}(\Delta)(\varepsilon)\left(x_{1} \wedge x_{2}\right) \wedge \mu^{-1}(\Delta)(\varepsilon)\left(x_{1} \vee x_{2}\right)$ $=\Delta(p(\varepsilon))\left(\mu\left(x_{1} \wedge x_{2}\right)\right) \wedge \Delta(p(\varepsilon))\left(\mu\left(x_{1} \vee x_{2}\right)\right)$ $=\Delta(p(\varepsilon))\left(\mu\left(x_{1}\right) \wedge \mu\left(x_{2}\right) \wedge \Delta(p(\varepsilon))\left(\mu\left(x_{1}\right) \vee \mu\left(x_{2}\right)\right)\right.$ $\geqslant \Delta(p(\varepsilon)) \mu\left(x_{1}\right) \wedge \Delta(p(\varepsilon)) \mu\left(x_{2}\right)$ $\geqslant \mu^{-1}(\Delta)(\varepsilon)\left(x_{1}\right) \wedge \mu^{-1}(\Delta)(\varepsilon)\left(x_{2}\right)$.

So, $f^{-1}(\Delta, \Omega)$ is a fuzzy soft lattice over $L_{1}$. 
Theorem 6 Let $S\left(L_{1}, E\right)$ and $S\left(L_{2}, E^{\prime}\right)$ be fuzzy soft classes over lattices $L_{1}$ and $L_{2}$, respectively, $p$ : $E \rightarrow E^{\prime}$ be an injection and $\mu$ be a homomorphic mapping from $L_{1}$ to $L_{2}$. If $(\Delta, \Omega) \in S\left(L_{1}, E\right)$, then $f(\Delta, \Omega) \in S\left(L_{2}, E^{\prime}\right)$.

Proof. For all $\beta \in p(E), y_{1}, y_{2} \in \mu\left(L_{1}\right)$, since $\mu$ is a homomorphic mapping, so there exist $x_{1} \in$ $\mu^{-1}\left(y_{1}\right), x_{2} \in \mu^{-1}\left(y_{2}\right)$ such that $x_{1} \wedge x_{2} \in \mu^{-1}\left(y_{1} \wedge\right.$ $\left.y_{2}\right), x_{1} \vee x_{2} \in \mu^{-1}\left(y_{1} \vee y_{2}\right)$. By Definition 20, $\mu(\Delta)(\beta)\left(y_{1} \wedge y_{2}\right)$

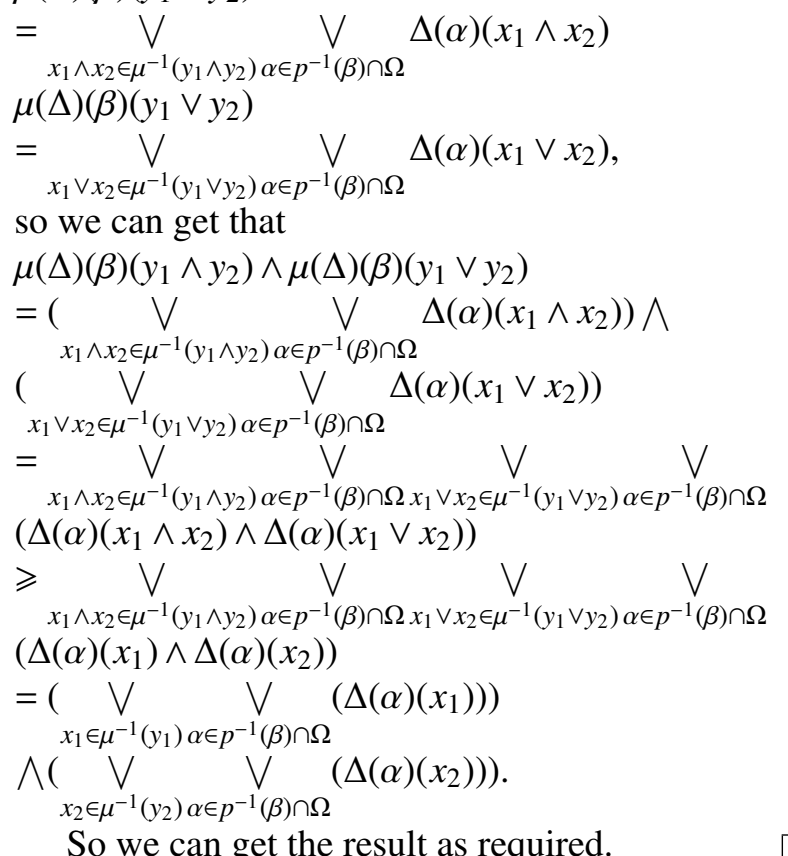

Theorem $7(S(L, E), \widetilde{\sqcup}, \widetilde{\Pi})$ forms a modular lattice.

Proof. By virtue of Proposition 4, Proposition 6 and Proposition 7, $(S(L, E), \widetilde{\sqcup}, \widetilde{\Pi})$ forms a lattice.

For modularity, let $\left(F_{i}, A_{i}\right) \in S(L, E),(i=1,2,3)$ be such that $\left(F_{1}, A_{1}\right) \geqslant\left(F_{2}, A_{2}\right)$. We have to prove that $\left(F_{1}, A_{1}\right) \sqcap\left(\left(F_{2}, A_{2}\right) \sqcup\left(F_{3}, A_{3}\right)\right)=\left(\left(F_{1}, A_{1}\right) \sqcap\right.$ $\left.\left(F_{3}, A_{3}\right)\right) \sqcup\left(F_{2}, A_{2}\right)$.

Since the inequality $\left(F_{1}, A_{1}\right) \sqcap\left(\left(F_{2}, A_{2}\right) \sqcup\right.$ $\left.\left(F_{3}, A_{3}\right)\right) \geqslant\left(\left(F_{1}, A_{1}\right) \sqcap\left(F_{3}, A_{3}\right)\right) \sqcup\left(F_{2}, A_{2}\right)$ is obvious, so it is enough to prove that $\left(F_{1}, A_{1}\right) \sqcap\left(\left(F_{2}, A_{2}\right) \sqcup\right.$ $\left.\left(F_{3}, A_{3}\right)\right) \leqslant\left(\left(F_{1}, A_{1}\right) \sqcap\left(F_{3}, A_{3}\right)\right) \sqcup\left(F_{2}, A_{2}\right)$.

Let

$\left(F_{1}, A_{1}\right) \sqcap\left(\left(F_{2}, A_{2}\right) \sqcup\left(F_{3}, A_{3}\right)\right)=(H, B),\left(\left(F_{1}, A_{1}\right) \sqcap\right.$ $\left.\left(F_{3}, A_{3}\right)\right) \sqcup\left(F_{2}, A_{2}\right)=(K, C)$. Firstly, since $\left(F_{1}, A_{1}\right) \geqslant$ $\left(F_{2}, A_{2}\right)$, so $A_{2} \subseteq A_{1}$, thus, it is clear that $A_{1} \cap\left(A_{2} \cup\right.$ $\left.A_{3}\right)=\left(A_{1} \cap A_{3}\right) \cup A_{2}$. Secondly, for all $\varepsilon \in A_{1} \cap\left(A_{2} \cup\right.$
$A_{3}$ ), we have that $\varepsilon \in A_{1}$ and $\varepsilon \in A_{2} \cup A_{3}$, (a) if $\varepsilon \in A_{2}, \varepsilon \notin A_{3}$, then, $H(\varepsilon)=F_{1}(\varepsilon) \sqcap F_{2}(\varepsilon)=$ $K(\varepsilon)$;

(b) if $\varepsilon \in A_{2}$ and $\varepsilon \in A_{3}$, then, $H(\varepsilon)=F_{1}(\varepsilon) \sqcap\left(F_{2}(\varepsilon) \sqcup\right.$ $\left.F_{3}(\varepsilon)\right) \subseteq F_{2}(\varepsilon) \sqcup F_{2}(\varepsilon) \sqcup F_{3}(\varepsilon)=\left(F_{1}(\varepsilon) \sqcap F_{2}(\varepsilon)\right) \sqcup$ $F_{3}(\varepsilon)=K(\varepsilon)$.

(c) if $\varepsilon \notin A_{2}$ and $\varepsilon \in A_{3}$, then, $H(\varepsilon)=F_{1}(\varepsilon) \sqcup F_{3}(\varepsilon)=$ $K(\varepsilon)$.

Hence, we can get the result as required. $\quad \square$ Let $(F, A)$ and $(G, B)$ be fuzzy soft lattices over $L$. We define $(F, A) \cup(G, B)=(H, C)$ as follows: $C=A \cap B$ and $H(\varepsilon)=F(\varepsilon) \sqcup G(\varepsilon)$ for all $\varepsilon \in C$. It is clear that we have the following:

Proposition 8 If $(F, A)$ and $(G, B)$ be fuzzy soft lattices over $L$, then $(H, C)=(F, A) \cup(G, B)$ is a fuzzy soft lattice over $L$.

Proposition 9 Let $(F, A),(G, B)$ and $(H, C)$ be fuzzy soft lattices over $L$. Then

1. $(F, A) \cup(F, A)=(F, A)$,

2. $(F, A) \uplus(G, B)=(G, B) \uplus(F, A)$,

3. $((F, A) \uplus(G, B)) \cup(H, C)=(F, A) \uplus((G, B) \uplus$ $(H, C))$.

Proposition 10 Let $(F, A),(G, B)$ be fuzzy soft lattices over L. Then

1. $((F, A) \cup(G, B)) \widetilde{\sqcap}(F, A)=(F, A)$,

2. $((F, A) \widetilde{\sqcap}(G, B)) \cup(F, A)=(F, A)$.

Theorem $8(S(L, E), \mathbb{U}, \widetilde{\Pi})$ is a modular lattice.

Definition 21 Let $(F, A)$ and $(E, B)$ be fuzzy soft lattices over $L$. We define $(F, A) \cap(E, B)=(H, C)$ as follows: $C=A \cap B$ and $H(\varepsilon)=F(\varepsilon) \cap E(\varepsilon)$ for all $\varepsilon \in C$.

It is clear that we have the followings:

Proposition 11 If $(F, A)$ and $(G, B)$ be fuzzy soft lattices over $L$, then $(H, C)=(F, A) \cap(G, B)$ is a fuzzy soft lattice over $L$.

Proposition 12 Let $(F, A),(G, B)$ and $(H, C)$ be fuzzy soft lattices over $L$. Then

1. $(F, A) \cap(F, A)=(F, A)$,

2. $(F, A) \cap(G, B)=(E, B) \cap(F, A)$,

3. $((F, A) \cap(G, B)) \cap(H, C)=(F, A) \cap((G, B) \cap$ $(H, C))$. 
Proof. Straightforward.

Similarly, we have the following propositions:

Theorem $9(S(L, E), \sqcup, \cap)$ is a modular lattice.

Proposition $13(S(L, E), \mathbb{U}, \cap)$ is a distributive lattice.

Proof. Similarly, we can get that $(S(L, E), \mathbb{U}$, ก $)$ is a distributive lattice. We only prove that $(S(L, E), \mathbb{U}, \cap)$ is distributive .

It is enough to prove that $(F, A) \cup((G, B)$ ก $(H, C))=((F, A) \cup(G, B))$ ก $((F, A) \cup(H, C))$ for all $(F, A),(G, B),(H, C) \in S(L, E)$.

Let $(F, A) \mathbb{U}((G, B) \cap(H, C))=(K, A \cap(B \cap C))$ and $((F, A) \cup(G, B)) \cap((F, A) \cup(H, C))=(M,(A \cap B) \cap$ $(A \cap B))=(M, A \cap B \cap C)$. For all $\varepsilon \in A \cap B \cap C$, that is, $\varepsilon \in A, \varepsilon \in B, \varepsilon \in C$, by Lemma 13, we can get that $K(\varepsilon)=F(\varepsilon) \sqcup(G(\varepsilon) \sqcap H(\varepsilon))=(F(\varepsilon) \sqcup$ $G(\varepsilon)) \sqcap(F(\varepsilon) \sqcup H(\varepsilon))=M(\varepsilon)$, thus, $(F, A) \cup((G, B) \cap$ $(H, C))=((F, A) \cup(G, B))$ ก $((F, A) \cup(H, C))$.

\section{Fuzzy soft ideal over $L$ and the lattice structure of fuzzy soft ideal over $L$}

Definition 22 Let $(F, A)$ be a fuzzy soft set over $L$, $(F, A)$ is called a fuzzy soft ideal if $F(\varepsilon)$ is a fuzzy ideal over $L$, for all $\varepsilon \in A$.

Theorem 10 Let $S(W, E)$ and $S\left(T, E^{\prime}\right)$ be fuzzy soft classes over lattices $W$ and $T$, respectively, $f$ : $S(W, E) \rightarrow S\left(T, E^{\prime}\right)$ be a mapping, $p: E \rightarrow E^{\prime}$ be a mapping and $\mu$ be a homomorphic mapping from $W$ to $T$. If $(\Delta, \Omega)$ is a fuzzy soft ideal over $T$, then $f^{-1}(\Delta, \Omega)$ is a fuzzy soft ideal over $S$.

Proof. For all $\alpha \in p^{-1}(\Omega), x_{1}, x_{2} \in \mu^{-1}(\Delta)(\alpha)$, we have that

$$
\begin{aligned}
& \mu^{-1}(\Delta)(\alpha)\left(x_{1} \vee x_{2}\right)=\Delta(p(\alpha))\left(\mu\left(x_{1} \vee x_{2}\right)\right) \\
& =\Delta(p(\alpha))\left(\mu\left(x_{1}\right) \vee \mu\left(x_{2}\right)\right) \\
& =\Delta(p(\alpha))\left(\mu\left(x_{1}\right)\right) \wedge \Delta(p(\alpha))\left(\mu\left(x_{2}\right)\right) \\
& =\mu^{-1}(\Delta)(\alpha)\left(x_{1}\right) \wedge \mu^{-1}(\Delta)(\alpha)\left(x_{2}\right) .
\end{aligned}
$$

Theorem 11 Let $S(W, E)$ and $S\left(T, E^{\prime}\right)$ be fuzzy soft classes over lattices $W$ and $T$, respectively, $f$ : $S(W, E) \rightarrow S\left(T, E^{\prime}\right)$ be a mapping, $p: E \rightarrow E^{\prime}$ be a mapping and $\mu$ be a surjective homomorphic mapping from $W$ to $T$. If $(\Delta, \Omega)$ is a fuzzy soft ideal over $W$, then $f(\Delta, \Omega)$ is a fuzzy soft ideal over $T$.
Proof. For all $\alpha \in p(\Omega), x_{1}, x_{2} \in \mu(\Delta)(\alpha)$, we have that

$$
\begin{aligned}
& \mu(\Delta)(\alpha)\left(x_{1} \vee x_{2}\right)=\Delta(p(\alpha))\left(\mu\left(x_{1} \vee x_{2}\right)\right) \\
& =\Delta(p(\alpha))\left(\mu\left(x_{1}\right) \vee \mu\left(x_{2}\right)\right) \\
& =\Delta(p(\alpha))\left(\mu\left(x_{1}\right)\right) \wedge \Delta(p(\alpha))\left(\mu\left(x_{2}\right)\right) \\
& =\mu(\Delta)(\alpha)\left(x_{1}\right) \wedge \mu(\Delta)(\alpha)\left(x_{2}\right)
\end{aligned}
$$

Theorem 12 Let $\mu_{1}, \mu_{2}$ be fuzzy ideals of $L$, then $\mu_{1} \sqcup \mu_{2}$ is a fuzzy ideal of $L$, where, $\mu_{1} \sqcup \mu_{2}$ is defined by $\left(\mu_{1} \sqcup \mu_{2}\right)(x)=\max \left\{\mu_{1}(x), \mu_{2}(x)\right\}$,

Proof. For all $x \in L, y \in L$, by Proposition 2 , if $x \leqslant y$, then $\mu_{1}(x) \geqslant \mu_{1}(y), \mu_{2}(x) \geqslant \mu_{2}(y)$, so we have that $\left(\mu_{1} \sqcup \mu_{2}\right)(x)=\max \left\{\mu_{1}(x), \mu_{2}(x)\right\} \geqslant$ $\max \left\{\mu_{1}(y), \mu_{2}(y)\right\}=\left(\mu_{1} \sqcup \mu_{2}\right)(y)$, so we can get that $\mu_{1} \sqcup \mu_{2}$ is a fuzzy ideal of $L$.

Lemma 14 Let $\mu_{1}, \mu_{2}$ and $\mu_{3}$ be fuzzy ideals of $L$, then

$$
\begin{aligned}
& \text { 1. } \mu_{1} \sqcup \mu_{1}=\mu_{1} \text {, } \\
& \text { 2. } \mu_{1} \sqcup \mu_{2}=\mu_{2} \sqcup \mu_{1} \text {, } \\
& \text { 3. }\left(\mu_{1} \sqcup \mu_{2}\right) \sqcup \mu_{3}=\mu_{1} \sqcup\left(\mu_{2} \sqcup \mu_{3}\right) \text {. }
\end{aligned}
$$

Proof. Straightforward.

Theorem 13 Let $\mu_{1}, \mu_{2}$ be fuzzy ideals of $L$, then $\mu_{1} \sqcap \mu_{2}$ is a fuzzy ideal of $L$, where, $\mu_{1} \sqcap \mu_{2}$ is defined by $\left(\mu_{1} \sqcap \mu_{2}\right)(x)=\min \left\{\mu_{1}(x), \mu_{2}(x)\right\}$,

Proof. It is similar to the proof of Theorem 12.

Lemma 15 Let $\mu_{1}, \mu_{2}$ and $\mu_{3}$ be fuzzy ideals of $L$, then

$$
\begin{aligned}
& \text { 1. } \mu_{1} \sqcap \mu_{1}=\mu_{1}, \\
& \text { 2. } \mu_{1} \sqcap \mu_{2}=\mu_{2} \sqcap \mu_{1}, \\
& \text { 3. }\left(\mu_{1} \sqcap \mu_{2}\right) \sqcap \mu_{3}=\mu_{1} \sqcap\left(\mu_{2} \sqcap \mu_{3}\right) .
\end{aligned}
$$

Proof. Straightforward.

Lemma 16 Let $\mu_{1}$ and $\mu_{2}$ be fuzzy ideals of $L$, then

$$
\begin{aligned}
& \text { 1. }\left(\mu_{1} \sqcap \mu_{2}\right) \sqcup \mu_{1}=\mu_{1} \text {, } \\
& \text { 2. }\left(\mu_{1} \sqcup \mu_{2}\right) \sqcap \mu_{1}=\mu_{1} \text {. }
\end{aligned}
$$

Proof. Straightforward.

Lemma 17 Let $\mu_{1}, \mu_{2}$ and $\mu_{3}$ be fuzzy ideals of $L$, then 


\section{1. $\left(\mu_{1} \sqcap \mu_{2}\right) \sqcup \mu_{3}=\left(\mu_{1} \sqcup \mu_{3}\right) \sqcap\left(\mu_{2} \sqcup \mu_{3}\right)$;}

2. $\left(\mu_{1} \sqcup \mu_{2}\right) \sqcap \mu_{3}=\left(\mu_{1} \sqcap \mu_{3}\right) \sqcup\left(\mu_{2} \sqcap \mu_{3}\right)$.

Proof. We only prove (1).

For all $x \in L$, without loss of generality, suppose that $\mu_{1}(x) \geqslant \mu_{2}(x)$, so we have that

$\left(\left(\mu_{1} \sqcap \mu_{2}\right) \sqcup \mu_{3}\right)(x)$

$=\max \left\{\min \left\{\mu_{1}(x), \mu_{2}(x)\right\}, \mu_{3}(x)\right\}$

$=\max \left\{\mu_{2}(x), \mu_{3}(x)\right\},\left(\left(\mu_{1} \sqcup \mu_{3}\right) \sqcap\left(\mu_{2} \sqcup \mu_{3}\right)\right)(x)$

$=\min \left\{\max \left\{\mu_{1}(x), \mu_{3}(x)\right\}, \max \left\{\mu_{2}(x), \mu_{3}(x)\right\}\right\}$

$=\max \left\{\mu_{2}(x), \mu_{3}(x)\right\}$,

so we can get that $\left(\mu_{1} \sqcap \mu_{2}\right) \sqcup \mu_{3}=\left(\mu_{1} \sqcup \mu_{3}\right) \sqcap\left(\mu_{2} \sqcup\right.$ $\left.\mu_{3}\right)$.

We denote the set of all fuzzy ideals of $L$ by $\mathscr{F} \mathscr{I}(L)$, then

Theorem $14(\mathscr{F} \mathscr{I}(L), \sqcup, \sqcap)$ forms a distributive lattice.

Proof. It is immediate by Lemma 14, Lemma 15, Lemma 16 and Lemma 17.

Proposition 14 Let $(F, A)$ and $(G, B)$ be fuzzy soft ideals over $L$, then $(H, C)=(F, A) \widetilde{\sqcap}(G, B)$ is a fuzzy soft ideal over $L$, where, $(H, C)$ is defined by $C=$ $A \cup B$ and for all $\varepsilon \in C$,

$$
H(\varepsilon)=\left\{\begin{array}{lll}
F(\varepsilon), & \text { if } \varepsilon \in A-B, \\
G(\varepsilon), & \text { if } \varepsilon \in B-A, \\
F(\varepsilon) \sqcap G(\varepsilon), & \text { if } \varepsilon \in A \cap B .
\end{array}\right.
$$

Proof. Straightforward.

Lemma 18 Let $(F, A),(G, B)$ and $(H, C)$ be fuzzy soft ideals over $L$. Then

1. $(F, A) \widetilde{\sqcap}(F, A)=(F, A)$,

2. $(F, A) \widetilde{\sqcap}(G, B)=(G, B) \widetilde{\Pi}(F, A)$,

3. $((F, A) \widetilde{\sqcap}(G, B)) \widetilde{\Pi}(H, C)=(F, A) \widetilde{\Pi}((G, B) \widetilde{\Pi}(H, C))$.

Proof. Straightforward.

Proposition 15 Let $(F, A)$ and $(G, B)$ be fuzzy soft ideals over $L$, then $(H, C)=(F, A) \widetilde{\sqcup}(G, B)$ is a fuzzy soft ideal over $L$, where, $(H, C)$ is defined by $C=$ $A \cup B$ and for all $\varepsilon \in C$,

$$
H(\varepsilon)=\left\{\begin{array}{lll}
F(\varepsilon), & \text { if } \quad \varepsilon \in A-B, \\
G(\varepsilon), & \text { if } \varepsilon \in B-A, \\
F(\varepsilon) \sqcup G(\varepsilon), & \text { if } \varepsilon \in A \cap B .
\end{array}\right.
$$

Proof. Straightforward.

Lemma 19 Let $(F, A),(G, B)$ and $(H, C)$ be fuzzy soft ideals over $L$. Then

1. $(F, A) \widetilde{\sqcup}(F, A)=(F, A)$,

2. $(F, A) \widetilde{\sqcup}(G, B)=(G, B) \widetilde{\sqcup}(F, A)$,

3. $((F, A) \widetilde{\sqcup}(G, B)) \widetilde{\sqcup}(H, C)=(F, A) \widetilde{\sqcup}((G, B) \widetilde{\sqcup}(H, C))$.

Proof. Straightforward.

Lemma 20 Let $(F, A)$ and $(G, B)$ be fuzzy soft ideals over $L$. Then

1. $(F, A) \widetilde{\sqcup}((F, A) \widetilde{\sqcap}(G, B))=(F, A)$,

2. $(F, A) \widetilde{\sqcap}((F, A) \widetilde{\sqcup}(G, B))=(F, A)$.

Proof. Straightforward.

Lemma 21 Let $(F, A),(G, B)$ and $(H, C)$ be fuzzy ideals of $L$, then

$$
\begin{array}{ll}
\text { 1. } & ((F, A) \widetilde{\sqcap}(G, B)) \widetilde{\sqcup}(H, C) \\
\quad= & ((F, A) \widetilde{\sqcup}(H, C)) \widetilde{\sqcap}((G, B) \widetilde{\sqcup}(H, C)) ; \\
\text { 2. } & ((F, A) \widetilde{\sqcup}(G, B)) \widetilde{\sqcap}(H, C) \\
\quad= & ((F, A) \widetilde{\sqcap}(H, C)) \widetilde{\sqcup}((G, B) \widetilde{\Pi}(H, C)) .
\end{array}
$$

Proof. It is immediate by Lemma 17 .

We denote the set of all fuzzy soft ideals over $L$ by $S(I, E)$ and the parameter set by $E$, that is, $S(I, E)=\{(F, A) \mid A \subseteq E, F: A \rightarrow P(U)\}$.

Theorem $15(S(I, E), \widetilde{\sqcup}, \widetilde{\Pi})$ forms a distributive lattice.

Proof. It is immediate by Proposition 14, Lemma 18, Proposition 15, Lemma 19, lemma 20 and Lemma 21.

Similarly, we have the followings:

Proposition 16 Let $(F, A)$ and $(G, B)$ be fuzzy soft ideals over $L$, then $(H, C)=(F, A) \cap(G, B)$ is a fuzzy soft ideal over $L$, where, $(H, C)$ is defined by $C=$ $A \cap B$ and for all $\varepsilon \in C, H(\varepsilon)=F(\varepsilon) \sqcap G(\varepsilon)$

Proof. Straightforward. 
Proposition 17 Let $(F, A)$ and $(G, B)$ be fuzzy soft ideals over $L$, then $(H, C)=(F, A) \cup(G, B)$ is a fuzzy soft ideal over $L$, where, $(H, C)$ is defined by $C=$ $A \cap B$ and for all $\varepsilon \in C, H(\varepsilon)=F(\varepsilon) \sqcup G(\varepsilon)$

Proof. Straightforward.

Theorem $16(S(I, E), \mathbb{U}, \cap)$ forms a distributive lattice.

Proof. It is similar to the proof of Theorem 15.

Theorem 17 (S(I,E), ש, $\widetilde{\Pi})$ forms a distributive lattice.

Proof. It is similar to the proof of Theorem 15.

Theorem $18(S(I, E), \widetilde{\sqcup}, \cap)$ forms a distributive lattice.

Proof. It is similar to the proof of Theorem 15.

\section{5. conclusion}

In this paper we have introduced the concept of fuzzy soft lattice and fuzzy soft ideal over a lattice and studied some of their related properties and discussed their lattice structure. By duality, we can discuss the related notions and properties of fuzzy soft filter over a lattice. To extend this work, one can study the properties of soft sets in other algebraic structures and fields. In addition, based on these results, we can further probe the applications of fuzzy soft lattices.

\section{Acknowledgment}

This work has been supported by the National Natural Science Foundation of China (Grant No. 61175044) and the Fundamental Research Funds for the Central Universities of China (Grant No. SWJTU11ZT29).

\section{References}

1. D.Molodtsov, Soft set theory-First results, Comput. Math. Appl. 37(1999)19-31.

2. P.K.Maji, A.R.Roy, R.Biswas, An application of soft sets in a decision making problem, Comput.Math.Appl. 44(2002)1077-1083.
3. D.G.Chen, E.C.C.Tsang, D.S.Yeung, X.Z.Wang, The parameterization of soft sets and its applications, Comput.Math.Appl. 49(2005)757-763.

4. P.K.Maji, R.Biswas, A.R.Roy, Fuzzy soft sets, J.Fuzzy Math.9(3)(2001)589-602.

5. K.Qin, Z.Hong, On soft equality, Journal of Computational and Applied Mathematics, 234(2010)13471355.

6. H.Aktas, N.Cağman, Soft sets and soft groups, Inform.Sci.177(2007)2726-2735.

7. F.Feng, Y.B.Jun, X.Z.Zhao, Soft semirings, Comput.Math.Appl. 56(2008)2621-2628.

8. Q.M.Sun, Z.L.Zhang, J.Liu, Soft sets and soft modules, Lecture Ntes in Comput.Sci. 5009(2008)403409.

9. M.I.Ali, F.Feng, X.Liu, W.K.Min, M.Shabir, On some new operations in soft set theory, Comput.Math.Appl. 57(2009)1547-1553.

10. Y.B.Jun, Soft BCK/BCI-algebras, Comput.Math.Appl. 56(2008)1408-1413.

11. J.Zhan, Y.B.Jun, Soft BL-algebras based on fuzzy sets, Comput.Math.Appl. 59(2010)2037-2046.

12. L.A.Zadeh, Fuzzy sets, Inf. Control. 8(1965)338-353.

13. P. Majumdar, S.K.Samanta, Generalised fuzzy soft sets, Comput.Math.Appl. 59(2010)1425-1432.

14. A.R.Roy,P.K.Maji, A fuzzy soft set theoretic approach to decision making problems, Journal of Computational and Applied Mathematics 203(2007)412-418.

15. Z.Kong, L.Gao, L.Wang, S.Li, The normal parameter reduction of soft sets and its algorithm, Comput.Math.Appl. 56(2008)3029-3037.

16. Z.Kong, L.Gao, L.Wang, Comment on A fuzzy soft set theoretic approach to decision making problems, J.Comput.Appl.Math. 223(2009)540-3037.

17. P.Majumdar, S.K.Samanta, On similarity measure of fuzzy soft sets, in:Proc. of the International Conference on Soft Computing \& Intelligent Systems, ICSCIS-07, Jabalpur(India)27-29 Dec., 2007, pp.4044.

18. P.Majumdar, S.K.Samanta, Similarity measure of soft sets, New Math. Nat. Comput.4(1)(2008)1-12.

19. C.Yang, Fuzzy soft semigroups and fuzzy soft ideals, Comput.Math.Appl. 61(2011)255-261.

20. A.Aygünoğlu, H.Aygün, Introduction to fuzzy soft groups, Comput.Math.Appl. 58(2009)1279-1286.

21. G.Grätzer,General Lattice Theory, Academic Press. New York, San Francisco, 1978.

22. FuLi, Soft Lattices, Global Journal of Science Frontier Research. 10(4)(2010)157-159.

23. M.Marudai, V.Rajendran, New Construction of Fuzzy Soft Lattices, International Journal of Computer Applications. 23(1)(2011)33-38.

24. G.Birkhoff, Lattice theory, Trans. Amer. Math.Sco., New York, 1967.

25. M.Attallah, Completely fuzzy prime ideals of dis- 
tributive lattices, J.Fuzzy Math. 8(1)(2000)151-156.

26. A.A.Estaji, S.Khodaii, S.Bahrami, On rough set and fuzzy sublattice, Informa.Sci. (2011),doi:10.1016/j.ins.2011.04.043

27. X.Zhang, Fuzzy logic and its algebraic analysis, Science Press. 2008 (In Chinese). 